(C) 2016 IEEE. Personal use of this material is permitted. Permission from IEEE must be obtained for all other uses, in any current or future media, including reprinting/republishing this material for advertising or promotional purposes, creating new collective works, for resale or redistribution to servers or lists, or reuse of any copyrighted component of this work in other works. 


\title{
Multiobjective Optimization of Multi-Carrier Energy System using a combination of ANFIS and Genetic Algorithms
}

\author{
Konstantinos Kampouropoulos, Student Member, IEEE, Fabio Andrade, Member, IEEE, Enric Sala, \\ Student Member, IEEE, Antonio Garcia Espinosa, Member, IEEE and Luis Romeral, Member, IEEE
}

\begin{abstract}
This paper presents a novel method for the energy optimization of multi-carrier energy systems. The presented method combines an adaptive neuro-fuzzy inference system, to model and forecast the power demand of a plant, and a genetic algorithm to optimize its energy flow taking into account the dynamics of the system and the equipment's thermal inertias. The objective of the optimization algorithm is to satisfy the total power demand of the plant and to minimize a set of optimization criteria, formulated as energy usage, monetary cost and environmental cost. The presented method has been validated under real conditions in the car manufacturing plant of SEAT in Spain in the framework of an FP7 European research project.
\end{abstract}

Index Terms-Energy optimization, multicarrier systems, dynamic optimization, mixed-integer programming, multiobjective problem, energy hub, energy prediction, optimal control, manufacturing plants.

\section{NOMENCLATURE}

The sets, indices, subscripts, variables, and parameters used in the equations throughout this paper are presented here.

\section{Indices}

$\begin{array}{ll}\alpha & \text { Index of hub's input energy carriers }[1: A] . \\ \beta & \text { Index for hub's output energy carriers }[1: B] . \\ t & \text { Index for optimization time instances }[1: T] . \\ i & \text { Index for energy converter types }[1: I] . \\ j & \text { Index for optimization criteria }[1: O] . \\ k & \text { Index for the number of installed components } \\ & \text { within the hub }[1: K] . \\ y & \text { Set of continuous optimization variables. } \\ y & \text { Set of discrete optimization variables. }\end{array}$

\section{Functions}

$g$

Equality constraint functions.

This work was supported in part by the Seventh Framework Programme under the FP7-ICT-2011-7 / 288102 Research Project EuroEnergest.

The authors are with the Fundació CTM Centre Tecnològic, Manresa, Spain, the University of Puerto Rico, Mayagüez, USA and the MCIA Research Center, Universitat Politècnica de Catalunya, Terrassa, Spain (email: konstantinos.kampouropoulos@ctm.com.es).

$\begin{array}{ll}h & \text { Inequality constraint functions. } \\ f & \text { Multiobjective optimization function. } \\ f_{j}^{\text {max }} & \text { Maximum objective function value of criterion } j . \\ f_{j}^{\text {trans }} & \begin{array}{l}\text { Transformed objective function value of } \\ \text { criterion } j .\end{array} \\ f_{j}^{o} & \text { Utopia point of objective function } j .\end{array}$

Parameters

$T \quad$ Number of time instances of the optimization horizon.

$C_{i}^{\beta \alpha} \quad$ Efficiency of the hub's energy converter of type $i$, for converting energy carrier $\alpha$ to energy carrier $\beta$.

$P_{i}^{i n} \quad$ Minimum input power for the energy converter of type $i$.

$\bar{P}_{i}^{i n} \quad$ Maximum input power for the energy converter of type $i$.

$\underline{P}_{T o t}^{i n, \alpha} \quad$ Minimum supply limit of energy carrier $\alpha$.

$\bar{P}_{\text {Tot }}^{\text {in } \alpha} \quad$ Maximum supply limit of energy carrier $\alpha$.

$L_{\beta} \quad$ Total output load of the hub of energy carrier $\beta$.

$\lambda_{t}^{\alpha} \quad$ Energy price of energy carrier $\alpha$ at the time instance $t$.

Variables

$P_{\text {Tot }}^{\text {in }} \quad$ Total input power of the hub.

$P_{\text {Tot }}^{\text {out }} \quad$ Total output power of the hub.

$P_{i k}^{i n} \quad$ Input power of the $\mathrm{k}^{\text {th }}$ energy converter of type $i$.

$P_{i k}^{\text {out }} \quad$ Output power of the $\mathrm{k}^{\text {th }}$ energy converter of type $i$.

$e^{\alpha} \quad$ Emission factor of the input energy carrier $\alpha$. 


\section{INTRODUCTION}

\section{A. Motivation and Approach}

$\mathrm{J}_{\mathrm{N}}$ recent years, the production and usage of energy has I become a topic of interest in the research community, in part because of environmental consciousness and support by governmental strategies, such as the EU 20/20/2020 climate and energy package which aims to convert Europe to a highly energy-efficient and low carbon economy [1]. One of the recent challenges that needs to be developed is the control of multicarrier energy systems composed by heterogeneous sources with different characteristics, in which there is a main focus in the study of electric systems, but other energy types suffer from reduced attention [2].

The Energy Hub (EH) is a recent concept that introduces a new paradigm for future multicarrier energy systems. Its key idea is to link different energy sources using current energy infrastructures such as energy converters and storage in an optimal manner [3]. This means that an EH effectively provides an interface between different sources and loads [4], offering important advantages, such as increased reliability of the system because of the implied redundancy, but also a gain in flexibility: it becomes possible to match the power demand by combining different sources. This flexibility permits to optimize the energy paths according to a global strategy, which can be of energetic, economic or of environmental character [5]-[7].

\section{B. Literature Review and Contributions}

The concept of the multicarrier energy systems, formulated as an energy hub structure was initially presented in [8]-[9]. Before that, several conceptual approaches for an integrated view of transmission and distribution systems with dispersed generation and storage have been published, such as "energyservices supply systems" [10], "basic units" [11] and "micro grids" [12]. There are few studies that discuss the hub design issue, while the majority are focused on the different operational issues in the multi-carrier energy systems, such as the economic dispatch [13]-[14], the optimal power flow [15][16], the unit commitment [17]-[18], and the optimal coupling of the energy carriers [19]. An approach in [20] considers the optimization of couplings among multiple energy networks consisting of electricity, natural gas and district-heating loads, while [21] presents a financial investment valuation method for energy hubs which includes conversion, storage and Demand Side Management capabilities (DSM). An integrated planning approach based on portfolio theory is discussed in [22], which calculates the optimal portfolio of energy supplies. In [23], a mixed-integer nonlinear programming problem is discussed, focused on the optimal coupling of the energy hub. This method is then extended in [24], evaluating the potential of connecting renewable energies into the system.

On the other hand, recent studies have focused on the integration of the EH in the framework of the Smart Grid, characterizing the concept of the Smart Energy Hubs (SEH) [25]. In this topic, a cloud-computing framework is presented in [26] that aims to resolve the problem of coordinating several SEH, in order to optimize the performance of the network. Furthermore, an integrated DSM technique is shown in [27], which models the interactions between different SEH as a non-cooperative game. Other studies of this topic propose and integrate a demand response program for SEH in order to modify the consumption patterns on the customer side [2829].

Nevertheless, the usage of the EH concept is mainly applied in real-time control applications which take decisions depending on data that are received from the plant, or in some cases, the state of the network. There is potential for improvement in this regard when taking into consideration the future status of the plant in two aspects: upcoming changes in power demand and the inertias of the equipment. Together these aspects may cause inefficiencies and instabilities to the system because of the EH's inability to entirely satisfy the energy demands, or due to operation decisions which result to unstable operation of the equipment.

In this paper, a new method for the optimization of multicarrier energy systems is proposed, which combines demand forecasting and nonlinear mixed-integer programming to tackle the problems caused by the dynamics of the system, taking into account both the future demand requirements and the inertias of the equipment. In this novel method, based on artificial intelligence algorithms, the operation of a multicarrier energy system is calculated for a future period of 24 hours, following the block diagram presented in Fig. 1. The

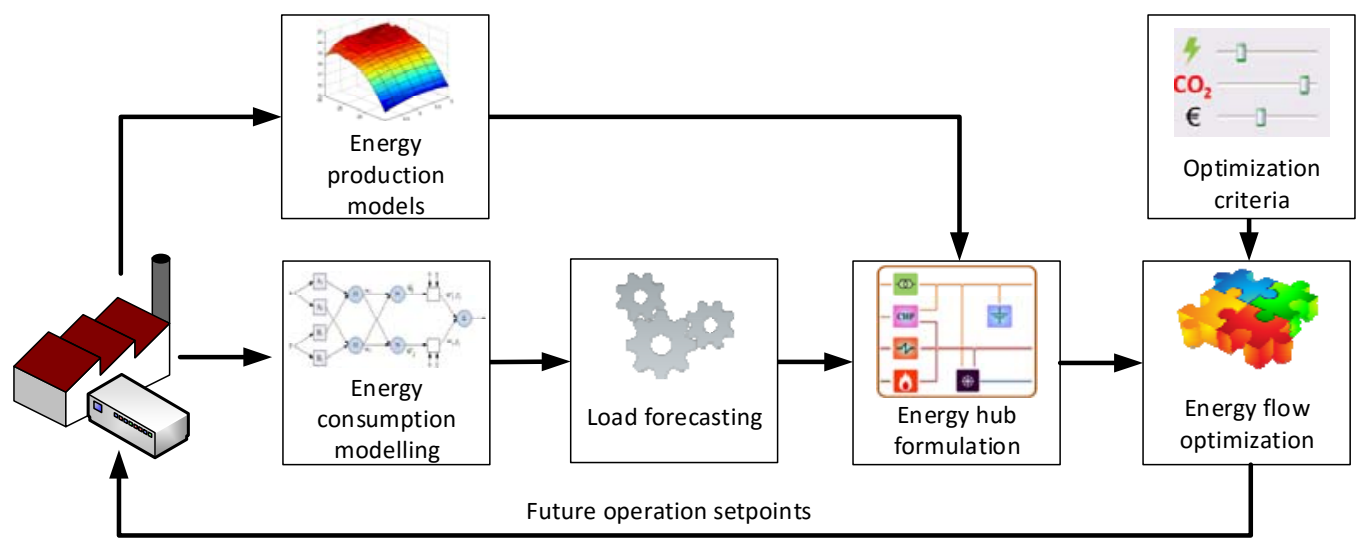

Fig. 1. Structure of the proposed optimization process. 
method consists on using a combination of Genetic Algorithms (GA) and Adaptive Neuro-Fuzzy Inference Systems (ANFIS) to model the energy profiles of the system and to predict the system's demands for a future range of 24 hours. Then, the energy flow of the system is optimized by means of a multiobjective GA with the aim to satisfy the energy demands, to minimize the total operation costs, the energy use and the generated $\mathrm{CO}_{2}$ emissions. Furthermore, the optimization process makes use of mathematical models of energy production equipment in order to take into account their inertias and time-responses. This information is used to update (if necessary) the operation bounds of the equipment in specific time instants, ensuring that the calculated solution is feasible and that no restrictions of the system are violated (e.g. operation of equipment out of bounds, exceed of energy sources, impractical operation strategies, etc.).

\section{Paper Structure}

This paper is organized as follows: Section II describes the mathematical formulation of the problem as well as the proposed optimization method. In Section III, the experimental validation and the numerical results are presented. Finally, Section IV presents the conclusions.

\section{OPTIMIZATION PROCESS}

This section describes the mathematical formulation of the optimization problem and provides a detailed presentation of the different steps of the proposed optimization process.

\section{A. Optimization problem formulation}

An energy hub can be considered as a system which contains multiple energy inputs and at least one output. Within the hub, energy can be converted to various forms in order to meet the load demand at its output ports. The energy transmission and conversion from an input hub port to an output hub is achieved through the available carriers. Based on this concept, different carriers can be employed in the hub in order to serve different loads or to offer alternative supplying paths. The objective of the hub problem is to satisfy the power demand of its outputs while minimizing (or maximizing) a set of optimization criteria for a future time period. In such a case, the optimization problem can be considered as a multi-period mixed integer problem and it can be formulated as:

$$
\begin{gathered}
\min \sum_{t=1}^{T} \sum_{j=1}^{O} f_{j}^{\text {trans }, t}(x, y) \\
\text { s.t. } g_{m}^{t}(x, y)=0 \\
\quad h_{m}^{t}(x, y) \leq 0
\end{gathered}
$$

where

$$
x=\left(x_{1}, \ldots, x_{N_{x}}\right) \text { and } y=\left(y_{1}, \ldots, y_{N_{y}}\right)
$$

$$
\begin{aligned}
& x \in S \subset \mathfrak{R}^{N_{x}}, \quad y \in D^{N_{y}} \\
& S=\left\{x \mid x_{n}^{l o} \leq x_{n} \leq x_{n}^{u p} \quad\left(n=1, \ldots, N_{x}\right)\right\} \\
& D=\left\{d_{1}, d_{2}, \ldots, d_{\max }\right\}
\end{aligned}
$$

In contrast to single-objective optimization, in multiobjective optimization the fitness function has to take into account all the objectives, which is done by using different assignment strategies, such as for instance the Pareto optimality. Among the different strategies, one of the general and most common scalarization methods for multi-objective optimization problems is the weighted global criterion method, in which all the objective functions are combined to form a single one. In order to sum the results of each criterion, a unit normalization is used. The most robust approach to transforming objective functions, regardless of their original range is given as follows [30]:

$$
f_{j}^{\text {trans }}=\frac{f_{j}(x, y)-f_{j}^{o}}{f_{j}^{\max }-f_{j}^{o}}
$$

In this paper the optimization objective of the hub's operation problem consists of multiple criteria at the same level of importance, which as presented in (9) to (11) are the minimization of the primary energy use of the hub, the minimization of the total cost for satisfying the energy demands and the minimization of the generated $\mathrm{CO}_{2}$ emissions.

$$
\begin{aligned}
& f_{1}^{t}=\sum_{\alpha} \sum_{t} P_{T o t, t}^{i n, a} \\
& f_{2}^{t}=\sum_{\alpha} \sum_{t} P_{T o t, t}^{i n, a} \cdot \lambda_{t}^{\alpha} \\
& f_{3}^{t}=\sum_{\alpha} \sum_{t} P_{T o t, t}^{i n, a} \cdot e^{a}
\end{aligned}
$$

Combining (9)-(11), the objective function of the problem is formulated as:

$$
F(x)=f_{1}^{\text {trans }} \cdot w_{1}+f_{2}^{\text {trans }} \cdot w_{2}+f_{3}^{\text {trans }} \cdot w_{3}
$$

where as $w$ are defined the weighted coefficients of the objectives, which in this case, are all equal to 1 .

\section{B. Proposed method}

The optimization process, as presented in Fig. 2, consists of 8 different steps which can be classified in 3 main blocks: the demand prediction for a future period of 24 hours; the mathematical formulation of the problem taking into account the conditions and restrictions of the system; and finally, the calculation of the optimum energy flow to satisfy the energy demands. The detailed characteristics of the method's steps are discussed next. 
1) Demand Modelling and Prediction: This task is performed by means of a combination of an ANFIS of TakagiSugeno type [31] and GA with objective to train the mathematical models of the system's demands, referenced to historical operation conditions and external parameters (e.g. climate conditions, time, etc.). This process is applied individually for each one of the system demands (i.e. outputs) and it uses two data sets in each case; a training set and a checking set that correspond to $70 \%$ and $30 \%$ of the available data, respectively. In the modelling process, the GA generates several training input combinations for the model, while the ANFIS algorithm calculates the relationships between the selected inputs and output, and generates the mathematical model. The obtained model is then evaluated by use of the checking data set, calculating an energy prediction vector and comparing it with the real measured data. The verification of the model's precision is made by means of the root mean square error (RMSE) and the symmetric mean absolute percentage error (SMAPE).

The training and evaluation process is repeated for all the input combinations of the models and the most accurate ones are kept. Once all of the mathematical models are obtained, they are used by the same algorithm to forecast the energy demands of the system for the following 24 hours, with oneminute intervals (1440 time instants). In this step, current and future data for the model's inputs are used, obtained by different sources (e.g. input by a user, gathered from a weather service, etc.). A detailed description of the modelling and prediction process was presented in [32], discussing the obtained accuracy results and the advantages of the algorithm's implementation over other similar methods.

2) Demand Analysis and Restructuring: In this step, the algorithm analyses the total energetic requirements of the system for the complete prediction horizon (following 24 hours) and applies a descending sorting to the demand vectors. Thus, the vectors are converted from time-ordered to descending-ordered, permitting to evaluate initially the time instants that present the higher energetic needs, calculating the optimal operation of the equipment and their settling times. In this way, the algorithm analyses and evaluates each time instant individually but it takes into account the operation of the equipment at the rest of the evaluated time instants (past and future) in order to recalculate (if necessary) the equipment's operation bounds. The calculation of the thermal inertias is made by using dynamic simulation models, developed with commercial software Apros [33] by VTT. The models are based on mechanistic simulations, using physical dimensions of the processes and pipelines, as well as equipment-specific parameters. They utilize dynamic conservation equations of mass, energy and momentum to calculate flows, temperatures, concentrations and pressures in the systems. The obtained simulation results were compared with measured data from the real system and adequate adjustments were made in the model's structure and parameters to improve their accuracy. A detailed description of the equipment's modelling process is presented in [34], discussing the modelling structure, the mathematical formulation and the obtained accuracy. Nevertheless, the optimization method is not restricted to use mechanistic simulation models, but other type of models can be implemented by maintaining a defined input-output structure.

3) Coefficient of Performance Constraints: During this step, the algorithm calculates the coefficient of performance (COP) of the energy production equipment (which can be constants, or their values can vary depending on the equipment and its operation conditions), as well as the energy demand of the evaluated time instant, and it formulates the equality constrains. In the case of a variable COP, the calculation is made by use of mathematical models of the equipment, which characterize their operation in different states. In the case of static ones, their efficiency is constant, provided by technical data sheets. As expressed in (13), the equality constraints state the possible energy carriers between the primary energy sources and demands (hub inputs and outputs respectively).

$$
P_{i k, t}^{\text {out }, \beta}=P_{i k, t}^{i n, \alpha} \cdot C_{i, t}^{\beta \alpha} \quad \forall \alpha, \forall \beta, \forall i, \forall k, \forall t
$$

4) Energy Supply Constraints: On the fourth step, the algorithm calculates the restrictions of the system and formulates the inequality constraint vectors. These restrictions can vary depending on the evaluated time instant, and as shown in (14) and (15), are related to the maximum available supply limits of the primary energies and the maximum limits of energy production by equipment.

$$
\begin{array}{lr}
\sum_{i} \sum_{k} P_{i k, t}^{i n, \alpha} \leq \bar{P}_{\text {Tot }, t}^{\text {in }, \alpha} & \forall \alpha, \forall i, \forall k, \forall t \\
\sum_{i} \sum_{k} P_{i k, t}^{\text {out }, \beta} / C_{i, t}^{\beta \alpha} \leq \bar{P}_{i, t}^{i n} & \forall \alpha, \forall \beta, \forall i, \forall k, \forall t
\end{array}
$$

5) Equipment's Operation Bounds: Next step is focused on the creation of the equipment's operation bound vectors. The values of these bounds are affected by the dynamic response of the equipment, as inertias and delays could be present while reaching the desired energy outputs from their current operation status. Equation (16) presents the formulation of the equipment's operation bounds in relation to their production.

$$
\underline{P}_{i, t}^{i n, \alpha} \leq P_{i k, t}^{i n, \alpha} \leq \bar{P}_{i, t}^{i n, \alpha} \quad \forall \alpha, \forall i, \forall k, \forall t
$$

6) Optimization of Time Instant: On this step the algorithm calculates the optimal energy flow of the multicarrier energy system of the current time instant by means of the GA algorithm. Initially, the mixed-integer problem is solved for every criterion individually to obtain their minimum feasible points (utopia points) and their maximum values. Then the whole multiobjective problem is solved, normalizing the criteria values with the transforming approach of (8). Once the algorithm optimizes the operation of the time instant $\mathrm{t}\{\mathrm{t} \in \mathbb{N} \mid 1 \leq \mathrm{t} \leq 1440\}$, the mathematical models of the 
equipment are used to calculate the maximum and minimum output powers that each equipment can produce for the following and previous time instants. These outputs may vary from the minimum and nominal output powers of the equipment due to possible delays in their operation. Thus, the equipment bounds are recalculated (are reduced if necessary) for the time instants $[\mathrm{t}+1, \ldots, \mathrm{t}+\mathrm{n}]$ and $[\mathrm{t}-1, \ldots, \mathrm{t}-\mathrm{m}]$ (where $\mathrm{t}-\mathrm{m}$ and $\mathrm{t}+\mathrm{n}$ cannot exceed the values 1 and 1440, respectively) and are taken into account by the optimization algorithm during the evaluation of the following instants.

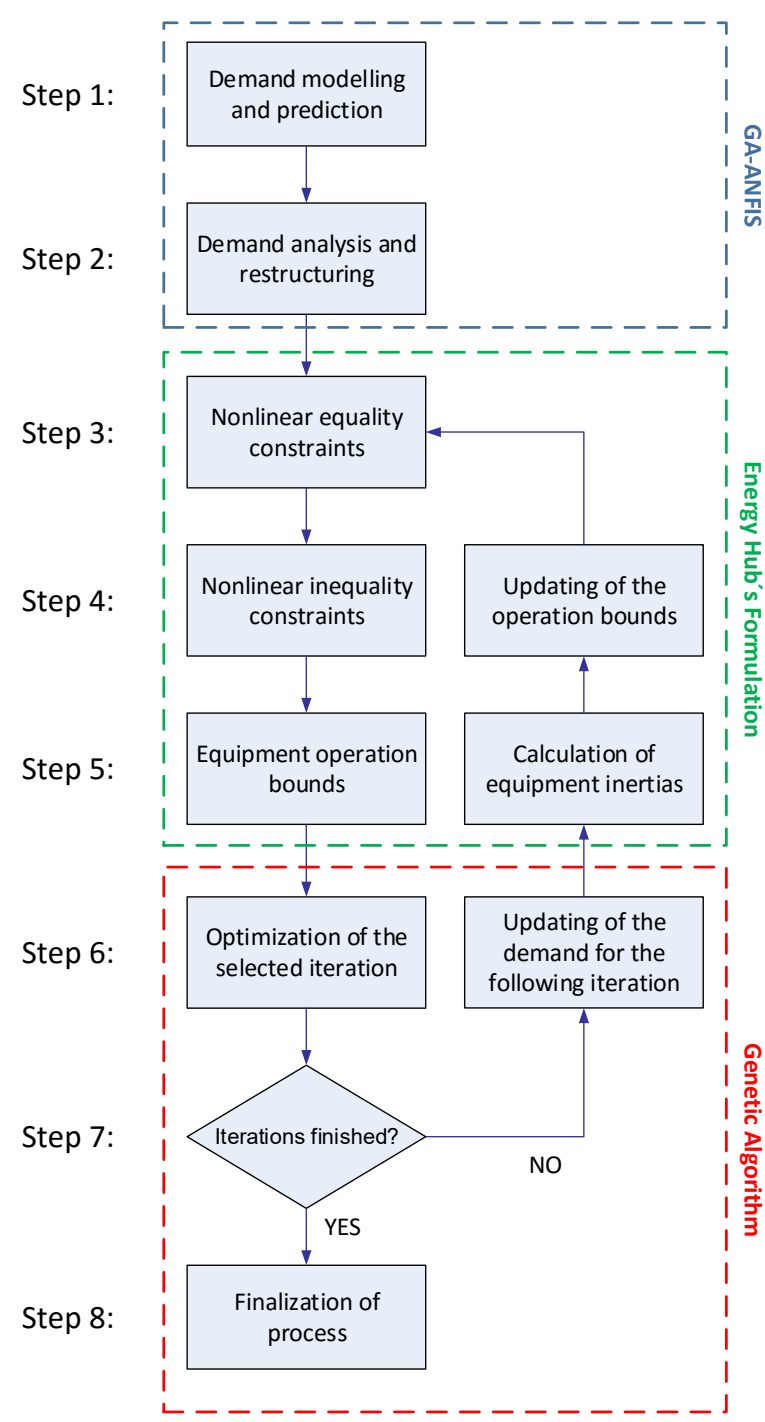

Fig. 2. Dataflow of the optimization process.

7) Evaluation and Updating of Conditions: In case that the optimization horizon has not yet evaluated completely, the algorithm continues to the next time instant, updating the entire problem's values (i.e. demands, constraints, bounds, prices and emissions). Furthermore, the updating of the necessary upper and lower equipment bounds is made, based on the calculated inertias from the previous time instants. Then steps 3 to 6 are repeated.

8) Normalization of Results: When all the optimization horizon is evaluated, the algorithm restores the results' order to the natural one (time-referenced) and provides the obtained optimal solution for the future period of 24 hours.

\section{IMPLEMENTATION AND RESULTS}

This section presents the validation results of the optimization method, proposed in Section II, applied in an automotive manufacturing plant in Spain. The evaluation and testing of the algorithm has been made in the framework of the FP7 European project EuroEnergest, using online production and consumption data, for a total duration of 3 months. The following subsections present the obtained results of the algorithm's implementation, compared with the normal operation of the automotive manufacturing plant for a period of 24 hours.

\section{A. System Overview}

A simplified structure of the validation multi-carrier energy plant is shown in Fig. 3. The plant includes two primary energy sources (i.e. electricity provided by the grid and natural gas provided by the network) and two types of energy demand (i.e. electric and heating). There exists a cogeneration equipment that consumes gas and produces heat and electricity, and three gas boilers for heating production. The power range of the equipment (individually) are in the range of tens of MW.

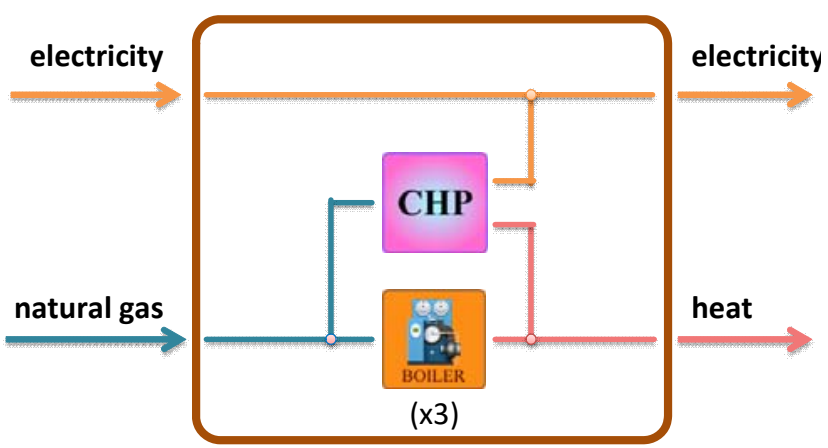

Fig. 3. Multi-carrier energy system structure.

The heat production $(Q)$ from the cogeneration machine and the gas boilers can be formulated as the product of the amount of natural gas consumed by each equipment and its thermal coefficient as described by the following equations.

$$
\begin{aligned}
& Q_{C H P}=\mathrm{C}_{C H P}^{\text {heat }} \cdot P_{\text {max }}^{\text {in gas }} \cdot f_{1} \quad, \quad 0 \leq f_{1} \leq 1 \\
& Q_{\text {Boiler } 1}=\mathrm{C}_{\text {Boiler } 1}^{\text {heat }} \cdot P_{\text {max }}^{\text {in gas }} \cdot f_{2} \quad, \quad 0 \leq f_{2} \leq 1 \\
& Q_{\text {Boiler 2 }}=\mathrm{C}_{\text {Boiler 2 }}^{\text {heat }} \cdot P_{\max }^{\text {in, gas }} \cdot f_{3} \quad, \quad 0 \leq f_{3} \leq 1 \\
& Q_{\text {Boiler 3 }}=\mathrm{C}_{\text {Boiler 3 }}^{\text {heat }} \cdot P_{\max }^{\text {in, gas }} \cdot f_{3} \quad, \quad 0 \leq f_{3} \leq 1
\end{aligned}
$$

The factors $f_{1}$ to $f_{4}$ represent the percentage of the natural gas use as input in the equipment, compared with the maximum source of gas $\left(P_{\max }^{\text {in } \text {,gas }}\right)$ supplied by the network. The sum of 
these factors cannot exceed the maximum quantity that can be supplied from the network. The amount of natural gas that is supplied to each equipment is calculated by the optimization algorithm during the evaluation of the different operation scenarios. The following equation describes the formulation of the system's restrictions in terms of energy supply availability (inequality constraints), combining (17)-(20). These restrictions are related to the maximum limit of natural gas use by the cogeneration and the three boilers.

$$
P_{\text {CHP }}^{\text {in gas }}+P_{\text {Boiler } 1}^{\text {in, gas }}+P_{\text {Boiler } 2}^{\text {in, gas }}+P_{\text {Boiler } 3}^{\text {in, gas }} \leq P_{\max }^{\text {in gas }}
$$

Finally, the electric power that is generated by the cogeneration depends on its thermal production and on the amount of its primary energy.

$$
P_{C H P}^{\text {elec }}=\mathrm{C}_{C H P}^{\text {elec }} \cdot P_{\max }^{\text {in gas }} \cdot f_{1} \quad, \quad 0 \leq f_{1} \leq 1
$$

The electric power that is supplied by the grid can be varied from zero to the maximum value of the contracted power (or the power that the installation of the plant supports). By combining (17)-(20) and (22), the available energy carriers inside of the plant can be formulated as shown in (23)-(24), expressing the interconnections between the system's outputs and inputs through the production equipment.

$$
\begin{aligned}
& L_{\text {elec }}=P_{\text {grid }}^{\text {in elec }}+P_{C H P}^{\text {in , gas }} \cdot C_{C H P}^{\text {elec }} \\
& L_{\text {heat }}=P_{C H P}^{\text {in, gas }} \cdot C_{C H P}^{\text {heat }}+P_{\text {Boiler 1 }}^{\text {in ,gas }} \cdot C_{\text {Boiler 1 }}^{\text {heat }}\left(d_{1}\right) \\
& +P_{\text {Boiler 2 }}^{\text {in, gas }} \cdot C_{\text {Boiler 2 }}^{\text {heat }}\left(d_{2}\right)+P_{\text {Boiler } 3}^{\text {in gas }} \cdot C_{\text {Boiler } 3}^{\text {heat }}\left(d_{3}\right)
\end{aligned}
$$

where

$$
d_{j} \in N \cap[0,4]
$$

As the operation of the boilers of this system is scalar (due to the existing control system that is implemented in the plant), the GA uses a discrete codified variable $d$ to define their operation status. This variable takes integer values between 0 and 4, which corresponds to an available produced output of the equipment (i.e. $0 \%, 25 \%, 50 \%, 75 \%$ and $100 \%$ respectively). Of course, more accuracy can be obtained by a finer discretization, but calculation burden is incremented. Depending on the selected operation point and based on their mathematical models, their coefficient of performance (COP) and their dynamic responses are calculated. These values are used during the evaluation of the operation scenario, as described in Step 6 of the proposed method.

\section{B. Optimization Results}

As described in Section II, the first step of the algorithm is the calculation of the system's energy demands by means of the GA-ANFIS algorithm. Figure 4 presents a comparison between the calculated predictions and the real values, for a validation period of 24 hours. The obtained models' accuracy errors are: $8.53 \%$ of RMSE and $4.31 \%$ of SMAPE for the electric demand, and $9.14 \%$ of RMSE and $5.12 \%$ of SMAPE for the heating demand respectively. Once the system's demands are obtained, the multiobjective optimization problem is formulated and solved for the considered time period.
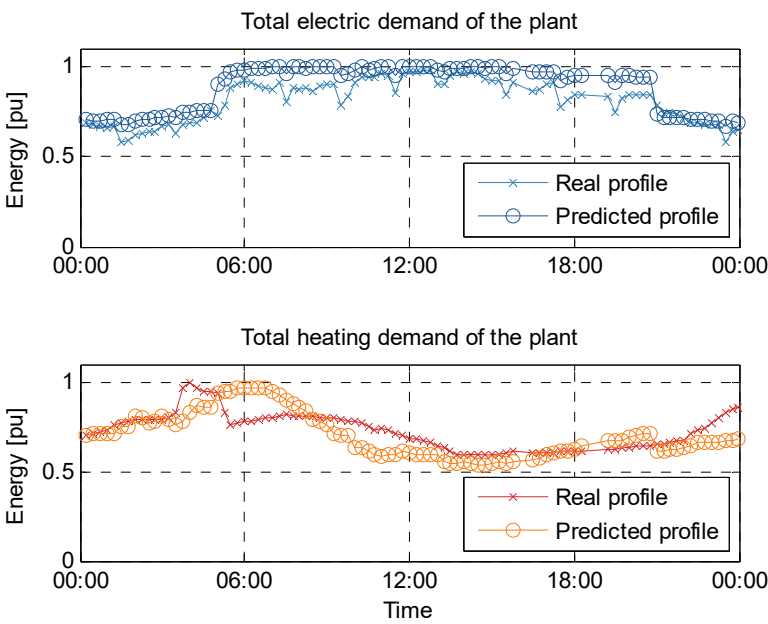

Fig. 4. Comparison between real demands and predictions.

As it was mentioned previously, during the evaluation of the feasible solutions, the algorithm takes into account the equipment's time response obtained by their models, which can affect their operation bounds. An example of the equipment's state models is presented in Fig. 5, which indicates the required time for the equipment to reach a desired operating point (setpoint) from its current one. The calculated times are then used as additional constraints in the problem's formulation in order to prevent all the unstable operation strategies. In the conventional operation of the system, the inertias are also being considered by the local controllers (PID of the equipment), but without taking into account the future profile of the demands, the variations of the energy prices during the day, or the equipment's efficiencies and interconnections.

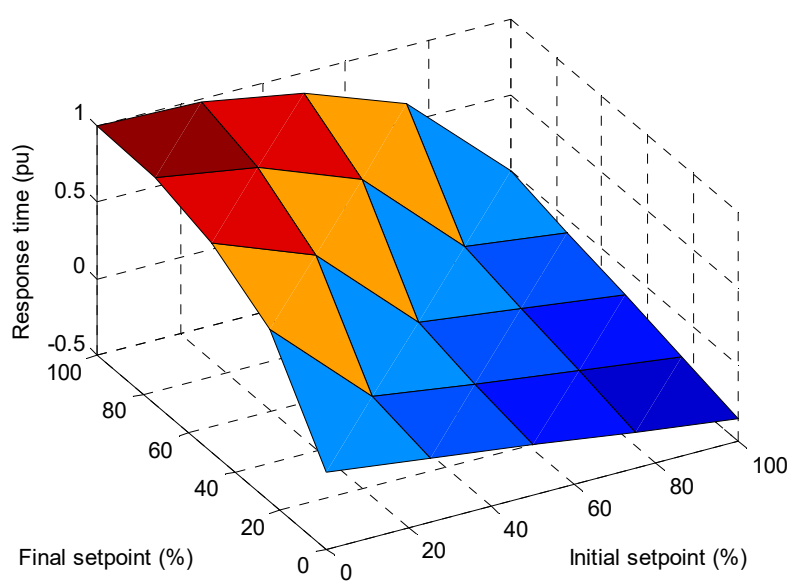

Fig. 5. Space state model of the boiler equipment. 
During this process, the algorithm calculates the optimal operation of the energy equipment which satisfies the energy demands and minimizes the optimization criteria which are defined in (9), (10) and (11). Fig. 6 presents the conventional (conv) and optimal operation (opt) of the energy production equipment for the whole optimization period of 24 hours. The information is presented in 15 minutes intervals.
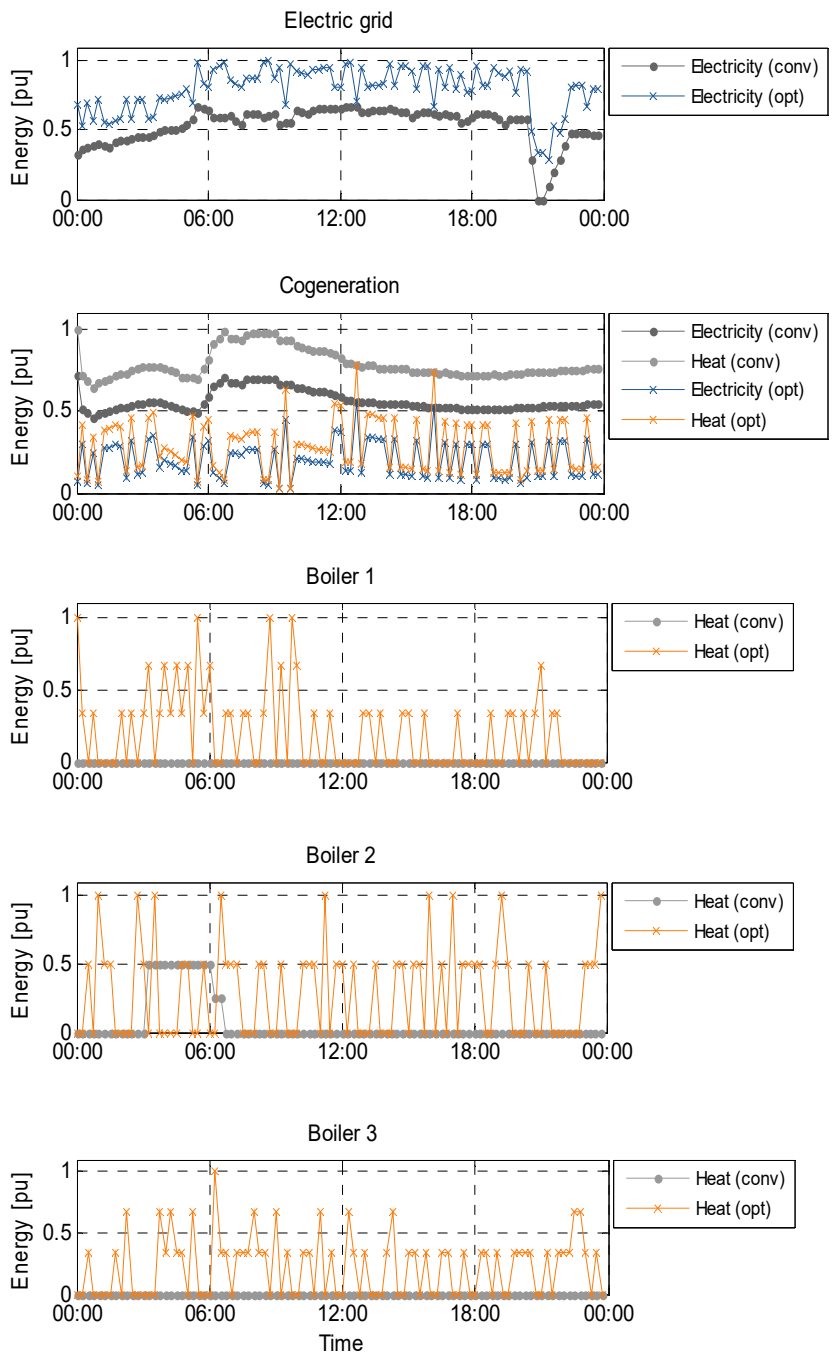

Fig. 6. Energy production per equipment.

A comparison between the optimal operation of the plant and the conventional one is shown in Fig. 7, indicating the energy amounts of electricity and gas, as well as the total energy production per equipment for the total period of 24 hours. It can be observed that the conventional operation strategy of the multi-carrier plant gives operation priority to the cogeneration equipment for the satisfaction of the heating demand (and partially the electric one), minimizing the use of the boilers and the grid's electric supply. However, the algorithm's solution presents a homogeneous distribution of effort between the equipment, reducing the consumption of natural gas. This occurs because of the higher performance value of the boilers in comparison to the cogeneration, which results in reduced amount of primary energy use for the fulfillment of the heating demand. Nevertheless, the consequence is the increment of the electric supply by the grid in order to satisfy the electric demand as well. This operation strategy may vary by considering different weight of the optimization criteria, or in cases in which the system has different energy rates through the day.

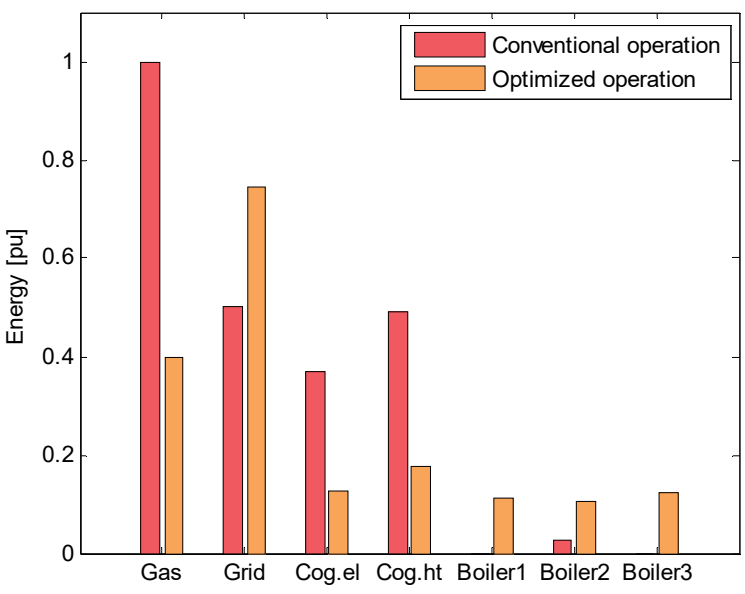

Fig. 7. Comparison of total energy use and production between conventional and optimized operation.

Finally, Fig. 8 shows a comparison of the optimized results between the two operations. It can be observed that the optimized operation of the system presents a minimization of the energy use by $33 \%$, a $22.7 \%$ of cost savings, and a $60.2 \%$ of $\mathrm{CO}_{2}$ emissions reduction. From the 3 months validation of the algorithm in real-site it was concluded an average potential savings of $16.24 \%$ of energy use, a $4.75 \%$ of energy cost decrement, and a $42.96 \%$ of generated emissions reduction.

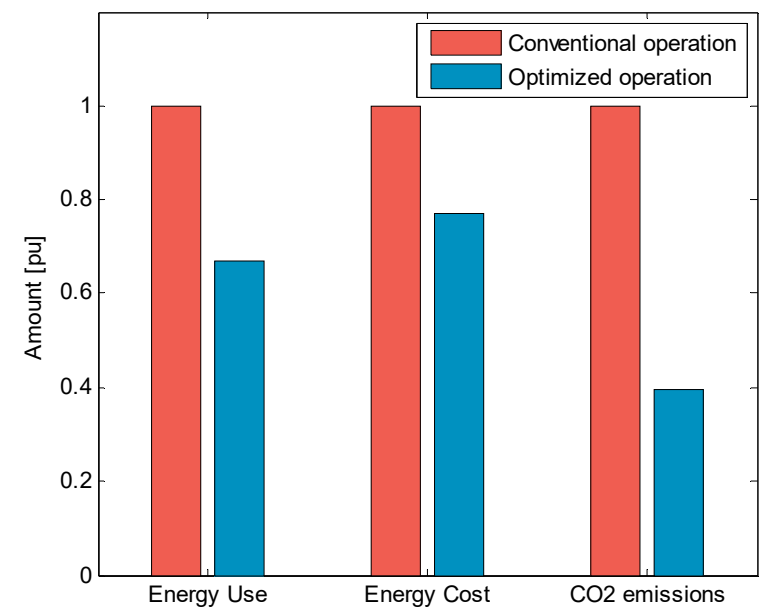

Fig. 8. Comparison of optimization criteria between conventional and optimized operation for a 24 hours period.

\section{CONCLUSIONS}

This paper presents a novel method in order to optimize the multi-period operation of multi-carrier energy systems by combining energy predictions and optimization into slot time 
periods, defined in a longer time horizon. It implements a combination of load forecasting algorithm (GA-ANFIS) and the energy hub concept in order to predict the energy demand of the system, while the optimization is carried out step by step into a predefined time horizon. The proposed method divides the optimization horizon into time slots, organized in a descending-order regarding the energetic requirements of each of them., The optimal operation of the energy production equipment and carriers for the higher demand situations are calculated initially, and it is guaranteed that the required demand of the system (even the peak demands) are entirely fulfilled. From this initial step, the following time slots are sequentially solved by simple non-dynamic optimization algorithms. Moreover, the method considers the dynamic responses of the system (inertias and delays on the equipment's operations), permitting to conclude to operation strategies that are stable for the system, while minimizing a set of optimization criteria. Finally, the required computational effort and time are reduced, due to the problem is being splitted and being resolved in multiple single-instants problems with no complex algorithms involved.

The proposed method has been tested and been validated under real conditions for a period of 3 months in the car manufacturing plant of SEAT in Martorell Spain, in the framework of the EuroEnergest FP7 European project. The experimental results present remarkable energetic, economic and environmental benefits by applying the proposed method to the validation plant.

Future work includes the consideration of energy storage technologies into the optimization method, in order to take advantages of the possible energy excess due to the equipment's inertias and use it in time instants of peak demands.

\section{REFERENCES}

[1] Communication from the Commission to the European Parliament, the Council, the European Economic and Social Committee and the Committee of the Regions; 2020 by 2020, Europe's climate change opportunity. Brussels, 23 Jan. 2008.

[2] Y. Dongmin, B. Lian, R. Dunn, and S. Le Blond, "Using control methods to model energy hub systems," 49th International Universities' Power Engineering Conference (UPEC), pp. 1-4, Sept. 2014.

[3] A. Shahmohammadi, M. Moradi-Dalvand, H. Ghasemi, and M.S Ghazizadeh, "Optimal Design of Multicarrier Energy Systems Considering Reliability Constraints," IEEE Transactions on Power Delivery, vol. 30, no. 2, pp. 878-886, Apr. 2015.

[4] M. Geidl, and G. Andersson, "Optimal Power Flow of Multiple Energy Carriers," IEEE Transactions on Power Systems, vol. 22, no. 1, pp. 145155, Feb. 2007.

[5] M. Geidl, G. Koeppel, P. Favre-Perrod, B. Klockl, G. Andersson, and K Frohlich, "Energy hubs for the future," IEEE Power Energy Mag., vol. 5, no. 1, pp. 24-30, Jan. 2007.

[6] M. Geidl, G. Koeppel, P. Favre-Perrod, B. Klöckl, G. Andersson, and K. Fröhlich, "The energy hub-A powerful concept for future energy systems," in Proc. 3rd Annu. Carnegie Mellon Conf. Elect. Ind., pp. 1314, Mar. 2007.

[7] K. Hemmes, J. Zachariah-Wolf, M. Geidl, and G. Andersson, "Towards multi-source multi-product energy systems," Int. J. Hydrogen Energy, vol. 32, pp. 1332-1338, 2007.

[8] R. Frik, and P. Favre-Perrod, "Proposal for a multifunctional energy bus and its interlink with generation and consumption," diploma thesis,
Power Systems and High Voltage Laboratories, ETH, Zurich, Switzerland, 2004.

[9] M. Geidl, "A greenfield approach for future power systems," in Proc. Cigre Session 41, Paris, France, 2006.

[10] H. M. Groscurth, T. Bruckner, and R. Kümmel, "Modeling of energy services supply systems," Energy, vol. 20, no. 9, pp. 941-958, 1995.

[11] I. Bouwmans, and K. Hemmes, "Optimising energy systems-Hydrogen and distributed generation," in Proc. 2nd Int. Symp. Distributed Generation: Power System Market Aspects, Stockholm, Sweden, 2002.

[12] R. H. Lasseter and P. Piagi, "Microgrid: A conceptual solution," in Proc. IEEE 35th Annu. Power Electronics Specialists Conf. (PESC), 2004.

[13] M. Moeini-Aghtaie, A. Abbaspour, M. Fotuhi-Firuzabad, and E. Hajipour, "Multiagent genetic algorithm: An online probabilistic view on economic dispatch of energy hubs constrained by wind availability," IEEE Trans. Sustain. Energy, vol. 5, no. 2, pp. 699-708, Apr. 2014.

[14] M. Geidl, and G. Andersson, "Optimal power dispatch and conversion in systems with multiple energy carriers," presented at the 15th Power Syst. Comput. Conf., 2005.

[15] A. Hajimiragha, C. Canizares, M. Fowler, M. Geidl, and G. Andersson, "Optimal energy flow of integrated energy systems with hydrogen economy considerations," in Proc. iREP Symp. Bulk Power Syst. Dynam. Control, pp. 1-11, 2007.

[16] M. Moeini-Aghtaie, A. Abbaspour, M. Fotuhi-Firuzabad, and E. Hajipour, "A decomposed solution to multiple-energy carriers optimal power flow," IEEE Trans. Power Syst., vol. 29, no. 2, pp. 707-716, Mar. 2014.

[17] L. Ramirez-Elizondo, V. Velez, and G. Paap, "A technique for unit commitment in multiple energy carrier systems with storage," in Proc. 9th Int. Conf. Environment Elect. Eng., pp. 106-109, 2010.

[18] L. Ramirez-Elizondo, and G. Paap, "Unit commitment in multiple energy carrier systems," in Proc. North Amer. Power Symp., pp. 1-6, 2009.

[19] M. Geidl, and G. Andersson, "Operational and topological optimization of multi-carrier energy systems," in Proc. Int. Conf. Future Power Syst., pp. 1-6, 2005.

[20] M. Geidl, and G. Andersson, "Operational and structural optimization of multi-carrier energy systems," Eur. Trans. Elect. Power, vol. 16, no. 5, pp. 463-477, 2006.

[21] F. Kienzle, P. Ahein, and G. Andersson, "Valuing investment in multienergy conversion storage, and demand-side management systems under uncertainty," IEEE Trans. Sustain. Energy, vol. 2, no. 2, pp. 194-202, Apr. 2011.

[22] F. Adamek, M. Arnold, and G. Andersson, "On decisive storage parameters for minimizing energy supply costs in multicarrier energy systems," IEEE Trans. Sustain. Energy, vol. 5, no. 1, pp. 102-109, Jan. 2014.

[23] M. Geidl and G. Andersson, "Optimal coupling of energy infrastructures," in Proc. IEEE Lausanne Power Tech. Conf., pp. 1398$1403,2007$.

[24] M. Schulze and P. C. Del Granado, "Optimization modeling in energy storage applied to a multi-carrier system," in Proc. Power Energy Soc. Gen. Meeting, pp. 1-7, 2010.

[25] M. Rayati, A. Sheikhi, and AM Ranjbar, "Optimizing operational cost of a smart energy hub, the reinforcement learning approach," International Journal of Parallel, Emergent and Distributed Systems, vol. 30, pp. 325341, Aug 2015.

[26] A. Sheikhi, M. Rayati, S. Bahrami, AM. Ranjbar, and S. Sattari, "A cloud computing framework on demand side management game in smart energy hubs," International Journal of Electrical Power \& Energy Systems, vol. 64, pp. 1007-1016, Jan 2015

[27] A. Sheikhi, M. Rayati, S. Bahrami, and AM. Ranjbar, "Integrated Demand Side Management Game in Smart Energy Hubs," IEEE Transactions on Smart Grid, vol. 6, no. 2, pp. 675-683, Mar 2015.

[28] S. Bahrami, and A. Sheikhi, "From Demand Response in Smart Grid Toward Integrated Demand Response in Smart Energy Hub," IEEE Transactions on Smart Grid, vol. PP, pp. 1-1, Aug. 2015.

[29] A. Sheikhi, S. Bahrami, and AM. Ranjbar, "An autonomous demand response program for electricity and natural gas networks in smart energy hubs," Energy, vol. 89, pp. 490-499, Sep 2015.

[30] R. T. Marler and J. S. Arora, "Survey of multi-objective optimization methods for engineering," Structural and Multidisciplinary Optimization, vol. 26, pp. 369-395, Apr 2004. 
[31] J. S. R. Jang, "ANFIS - Adaptive-network-based fuzzy inference system," IEEE Transactions on Systems Man and Cybernetics, vol. 23, May 1993.

[32] K. Kampouropoulos, J. J. Cardenas, F. Giacometto, and L. Romeral, “An energy prediction method using Adaptive Neuro-Fuzzy Inference System and Genetic Algorithms," IEEE International Symposium on Industrial Electronics (ISIE), pp. 1-6, May 2013.

[33] Apros.fi, "Apros - Dynamic process simulation software for nuclear and thermal power plant applications," Internet: www.apros.fi, Jan 2016.

[34] J. Savolainen, J. Lappalainen, A. Aikala, and P. Ruuska, "Enhancing energy management of a car manufacturing plant through modelling and dynamic simulation," 29th European Conference on Modelling and Simulation (ECMS), pp. 259-265, May 2015.

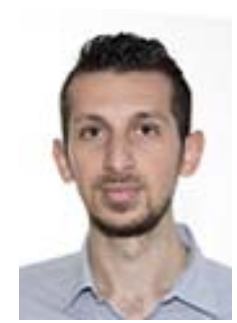

Konstantinos Kampouropoulos (S'12) received the electrical engineering degree from the Public Institution of Higher Education of Western Macedonia, Kozani, Greece, in 2009. He is currently studying for $\mathrm{a} \mathrm{PhD}$ at the Universitat Politècnica de Catalunya dealing with optimization algorithms and energy efficiency. ,Since 2008, he is a member of the Energy Area of Fundació CTM Centre Tecnològic, working with $\mathrm{I}+\mathrm{D}$ projects related to energy efficiency, energy optimization, maintenance processes and development of algorithms for the energy management.

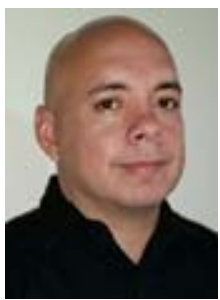

Fabio Andrade (M'15) received the Ph.D. degree from the Universitat Politècnica de Catalunya (UPC), Barcelona, Spain, in 2013, his B.Sc in electronic engineering and his Master degree in Engineering with emphasis on Automatic Control from the Universidad Del Valle, Cali, Colombia in 2004 and 2007, respectively. In 2009, he joined the Motion Control and Industrial Centre Innovation Electronics (MCIA). In 2014, He worked as a Postdoc researcher in UPC and Aalborg University, Denmark. Currently, he is working as an Assistant Professor on power electronics applied to renewable energy in the University of Puerto Rico, Mayaguez campus. His main research interests include modeling, analysis, design, and control of power electronic converters, principally for $\mathrm{dc} / \mathrm{ac}$ power conversion, grid-connection of renewable energy sources, and Microgrid application.

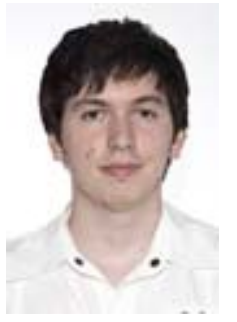

Enric Sala (S'14) received the M.S. degree in industrial electronics and automation from the Universitat Politècnica de Catalunya (UPC), Barcelona, Spain, in 2013. He is currently working toward the Ph.D. degree in Motion Control and Industrial Applications Center (MCIA), Universitat Politècnica de Catalunya, Barcelona, Spain.

His research interest include energy efficiency, advanced energy management strategies for smart buildings.

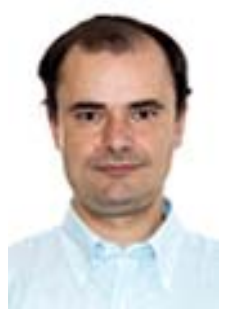

Antonio Garcia Espinosa (M'05) received the M.S degree in electrical engineering and the Ph.D. degree from the Universitat Politècnica de Catalunya (UPC), Barcelona, Spain, in 2000 and 2005, respectively.

Since 2000, he has been with the Electric Engineering Department, UPC, where he is currently a Lecturer. He is with the Motion and Industrial Control Group. His research interests include electromagnetic devices, electric machines, variablespeed drive systems, and fault-detection algorithms.

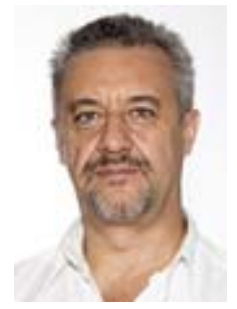

Luis Romeral (M'98) received the M.S. degree in electrical engineering and the Ph.D. degree from the Universitat Politècnica de Catalunya (UPC), Barcelona, Spain, in 1985 and 1995, respectively. In 1988, he joined the Electronic Engineering Department, UPC, where he is currently an Associate Professor and the Director of the Motion and Industrial Control Group (MCIA), whose major research activities concern induction and permanent magnet motor drives, enhanced efficiency drives, fault detection and diagnosis of electrical motor drives, and improvement of educational tools. He has developed and taught post-graduate courses on programmable logic controllers, electrical drives and motion control, and sensors and actuators. 